\title{
Implante intra-ocular de lentes dobráveis "piggyback" em paciente portador de catarata congênita no primeiro ano de vida - Relato de caso
}

\author{
Piggyback foldable intraocular lens implant in patient with congenital cataract in \\ the first year of life - Case report
}

\author{
Leonardo Akaishi ${ }^{1}$ \\ Rodrigo Vaz $^{2}$ \\ Virgínia Delacroix Cury ${ }^{3}$ \\ Sérgio Luiz Kniggendorf ${ }^{4}$
}

Patrick Frensel de Moraes Tzelikis ${ }^{5}$

\section{RESUMO}

No presente relato os autores descrevem um caso de catarata congênita unilateral tratada cirurgicamente com implante intra-ocular de lentes dobráveis "piggyback", ainda não descrito na literatura nacional. São analisadas as diferenças e semelhanças com outros casos descritos na literatura.

Descritores: Catarata/congênita; Extração de catarata; Lente intra-ocular; Implante de lente intra-ocular; Acuidade visual; Relato de caso

\section{INTRODUÇÃO}

Catarata congênita (CC) é uma importante causa de baixa visual moderada a severa na infância, sendo responsável por 15 a 20\% dos casos de cegueira em escolas para cegos nos Estados Unidos ${ }^{(1)}$. Estima-se que para cada 250 neonatos, um possua alguma forma de catarata congênita ${ }^{(2)}$. No Brasil, a CC apresenta uma incidência variando de 5,5\% a $12 \%$ em pacientes portadores de visão subnormal ${ }^{(3)}$.

O termo catarata congênita refere-se a opacidades presentes ao nascimento $^{(4)}$. Apesar do desenvolvimento de novas técnicas e materiais cirúrgicos a catarata congênita continua a ser um desafio para os oftalmologistas. Diferente da cirurgia do adulto, que proporciona excelentes resultados visuais, a CC possui muitas peculiaridades e controvérsias. Um dos poucos consensos é que a $\mathrm{CC}$ deve ser operada precocemente, de preferência antes das doze semanas de vida(1-2,5).

A lente intra-ocular (LIO) continua a ser motivo de grande controvérsia. Existem opiniões divergentes quanto à época ideal para o implante, quanto ao material da LIO a ser utilizada, e o poder da lente a ser implantada. Existem autores favoráveis ao implante somente após os dois anos de idade $^{(6)}$, entretanto outros advogam o implante mais precocemente ${ }^{(7)}$.

O implante de 2 ou mais lentes na câmara posterior (polipseudofacia ou "piggyback") foi introduzido por Gayton e Sanders em $1993^{(5)}$. Os objetivos de se utilizar lentes em "piggyback" são, principalmente, para atingir uma adequada correção óptica em pacientes que requerem altos graus de poder em lentes intra-oculares (LIO) e para permitir correção secundária de erro resultante de implante inadequado de LIO após cirurgia de catarata. Apesar de já ter sido referenciada na literatura internacional, este trabalho vem descrever pela primeira vez na literatura nacional um caso de catarata congênita unilateral tratada cirurgicamente com duplo implante intra-ocular "piggyback". Há pleno consentimento dos pais do menor, da comissão de 
ética do Hospital Oftalmológico de Brasília e da Sociedade Brasileira de Catarata e Implantes Intra-oculares que, consultada, não desaprova a realização deste procedimento.

\section{RELATO DE CASO}

J.M.C.M, 1 mês e 10 dias de vida, sexo masculino, leucodérmico, natural e residente em Brasília - DF. Foi encaminhado para avaliação no Hospital Oftalmológico de Brasília (HOB) com quadro de leucocoria no olho esquerdo (OE) detectada desde o nascimento. Pais saudáveis e sem história de consangüinidade. História gestacional sem intercorrências, parto natural, a termo, peso ao nascer de $3.165 \mathrm{~g}$.

Ao exame oftalmológico paciente não colaborava, não fixava nem seguia objetos em ambos os olhos (AO). Os reflexos pupilares estavam normais em AO.

À ectoscopia o OE apresentava uma leucocoria. O olho direito (OD) não apresentava alteração.

À biomicroscopia sob sedação observou-se no $\mathrm{OE}$, olho calmo, córnea transparente, câmara anterior média, íris eutrófica, e uma catarata total. A fundoscopia do OE era inviável. O exame biomicroscópico do OD bem como a fundoscopia eram normais. A pressão intra-ocular em ambos os olhos (AO) era de $8 \mathrm{mmHg}$. A ultra-sonografia do OE não apresentava alterações. O comprimento axial era de $18,48 \mathrm{~mm}$ no OD e $17,30 \mathrm{~mm}$ no OE. O cálculo do poder da LIO usando a fórmula Holladay II encontrou um valor de 46,5 dioptrias (D) para a emetropia. Foi escolhido uma lente com poder total de 43,00 D para correção.

$\mathrm{O}$ paciente foi então submetido à cirurgia de catarata. Sob anestesia geral, uma facoaspiração via córnea clara, temporalmente, com capsulorrexe anterior e posterior primária e vitrectomia anterior central foi realizada sem intercorrências. Foram implantadas dentro do saco capsular duas lentes dobráveis, sendo uma posterior de acrílico de 28,00 D (AcrySof ${ }^{\circledR}$ modelo SA30AT) e outra anterior de silicone de 15,00 D (Allergan ${ }^{\circledR}$ modelo SI40NB). No final realizou-se uma iridectomia periférica sub incisional (Figura 1).

No pós-operatório de um mês através de estímulo visual constante e utilizando tampão o paciente passou a apresentar fixação intermitente. Ao exame observou-se olho calmo, córnea transparente, câmara anterior média com poucas células, as LIOs bem posicionadas e uma discreta opacificação posterior. A retinoscopia foi encontrado $\mathrm{OD}=+4,50 \mathrm{DE}$ e no $\mathrm{OE}=$ $+5,50 \mathrm{DE}$.

No retorno do terceiro mês a opacificação posterior era maior e foi indicado vitrectomia posterior via pars plana e aspiração de pérolas de Elschning na interface das duas lentes (Figura 2).

No sexto mês pós-operatório, o paciente apresentava retinoscopia sob cicloplegia do $\mathrm{OD}=+3,00$ e do $\mathrm{OE}=$ plano. No nono mês pós-operatório a retinoscopia era de $\mathrm{OD}=+3,00 \mathrm{e}$ $\mathrm{OE}=-1,50$.

Atualmente o paciente encontra-se no décimo-quarto mês pós-operatório apresentando fixação ocular alternante, eixo visual livre, retinoscopia sob cicloplegia do $\mathrm{OD}=+1,50$ e do $\mathrm{OE}=-4,00 \approx-0,50 \times 140^{\circ}$. Apresenta excelente desenvolvimento neuro-psico-motor, estando sob constantes cuidados de uma equipe multidisciplinar na tentativa de combater a ambliopia.

\section{DISCUSSÃO}

O procedimento cirúrgico em $\mathrm{CC}$ requer que se leve em consideração uma série de fatores próprios desta patologia. $\mathrm{O}$ prognóstico visual no olho afetado em casos de CC unilateral depende da rápida restauração de um eixo visual transparente, um método de correção da afacia eficaz e um agressivo tratamento para ambliopia ${ }^{(8)}$.

Indica-se o procedimento cirúrgico em $\mathrm{CC}$ quando existe qualquer opacidade central e/ou cortical adjacente do cristalino, com distorção de mais de $3,0 \mathrm{~mm}$, que tem demonstrado ser visualmente significante ${ }^{(1)}$. Se com oclusão, e/ou dilatação pupilar, a acuidade visual for pior que $20 / 60$, provavelmente a cirurgia para extração da catarata também esteja indicada ${ }^{(2)}$. Nos casos unilaterais, ou bilaterais completos, presentes ao nascimento, onde as chances de ambliopia são maiores, a cirurgia está indicada o mais cedo possível ${ }^{(1-9)}$.

Diversas técnicas cirúrgicas para tratamento da $\mathrm{CC}$ já foram descritas, incluindo lensectomia e vitrectomia com implante de LIO na câmara anterior ${ }^{(10)}$, lensectomia associada a capsulotomia posterior e vitrectomia anterior ${ }^{(11-12)}$, facectomia extracapsular via límbica com capsulorrexe anterior e posterior primária ${ }^{(1)}$, entre outras, sendo que cada uma apresenta suas vantagens e desvantagens.

A LIO ainda é motivo de muita controvérsia na abordagem da CC. Existe hoje uma tendência mundial de implante primário de LIO em crianças independente da faixa etária ${ }^{(1-7)}$. O poder da LIO a ser implantada é de fundamental importância e depende do comprimento antero-posterior, ceratometria e profundidade da câmara anterior do olho. Deve-se levar em conta o crescimento ocular ${ }^{(11)}$. Estudos mostram que o comprimento axial no recém-nascido é de aproximadamente $17 \mathrm{~mm}$ e passam para 23,6 mm no adulto, portanto, a maior parte do crescimento ocular ocorre nos dois primeiros anos de vida, dificultando ainda mais a seleção da melhor LIO para implante. Alguns autores ${ }^{(13)}$ observaram que o crescimento axial, ao nascimento é de $17 \mathrm{~mm}$, depois aumenta de 3,5 $\mathrm{mm}$ no primeiro ano, de 0,9 $\mathrm{mm}$ no segundo ano e de $0,3 \mathrm{~mm}$ no terceiro ano. Desta forma deve-se ter cuidado no implante de LIO precocemente, visando a emetropia, pois o crescimento ocular pode resultar tardiamente em uma refração miópica acentuada.

Em decorrência do crescimento axial, o cálculo da LIO, principalmente em crianças menores de dois anos, ainda representa um importante problema a ser resolvido. McClatchey desenvolveu um programa de computador que objetiva prever o erro refracional final. Seu método utiliza as fórmulas SRK $2 \mathrm{e}$ Holladay I. Acredita-se que esta fórmula super-estime o poder da LIO necessária para olhos com diâmetro antero-posterior 


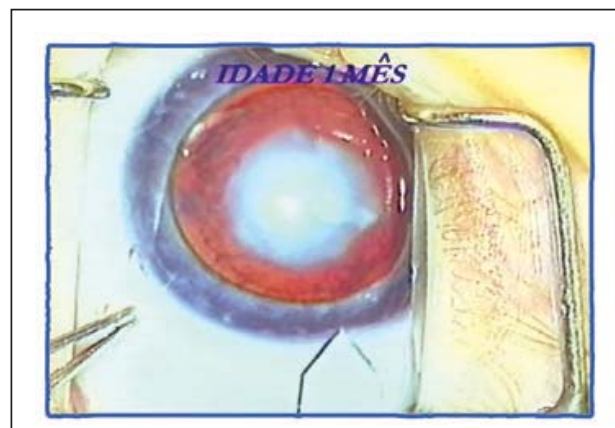

A

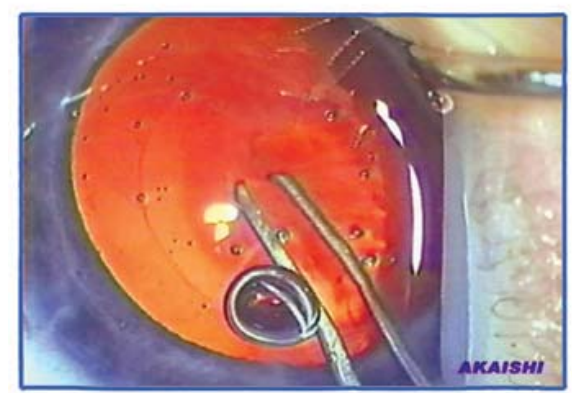

D

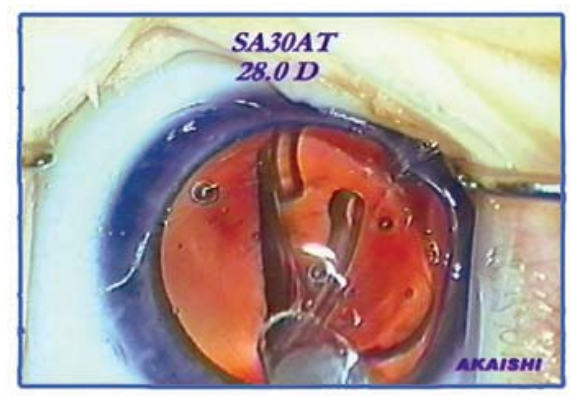

G

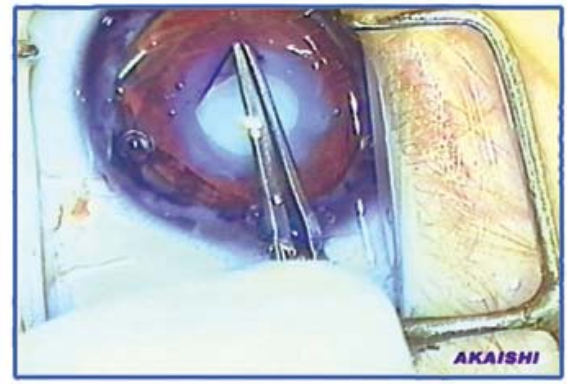

B

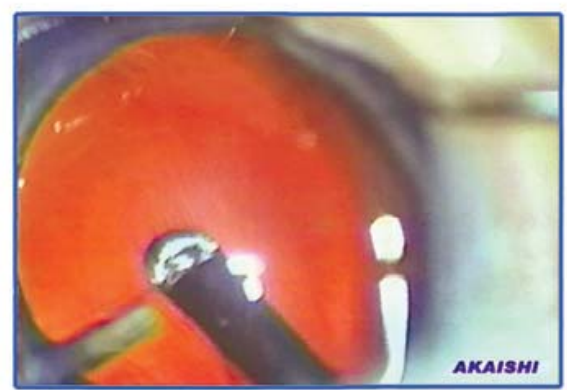

E

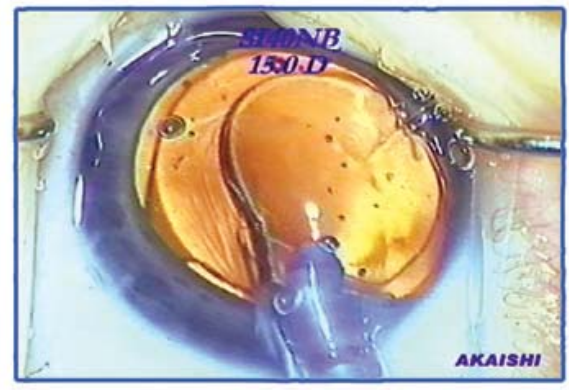

H

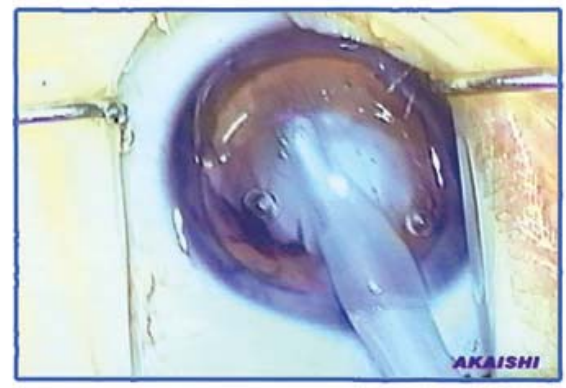

C

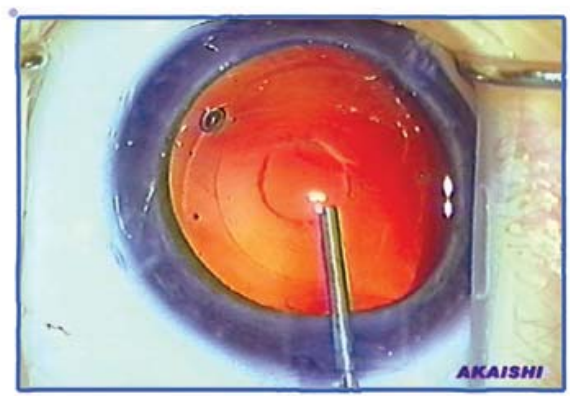

F

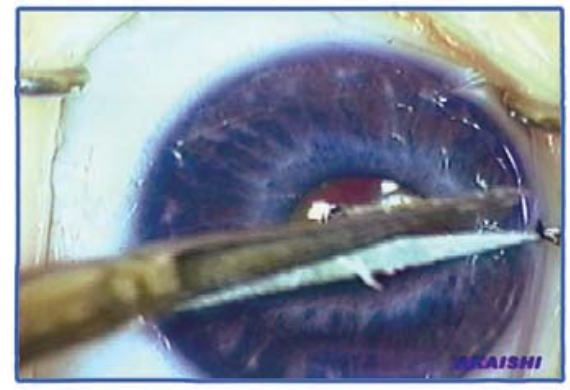

I

Figura 1 - (Akaishi) A: Incisão temporal em córnea transparente; B: Capsulorrexe com azul de tripan; C: Facoaspiração com caneta de l/A; D: Capsulorrexe posterior; E: Vitrectomia anterior; F: Injeção de viscoelástico para implante das LIOs; G: Implante da primeira LIO no saco capsular (SA30AT); H: Implante da segunda LIO no saco capsular (SI40NB); I: Iridectomia periférica, aspiração do viscoelástico e formação da câmara anterior com incisões auto-selantes

pequeno $^{(14)}$. No presente estudo, optamos por calcular o poder dióptrico da LIO utilizando a fórmula Holladay II que acreditamos ser mais exata para a escolha de lentes para olhos com diâmetro antero-posterior reduzido. Existem atualmente várias propostas para solução deste problema. Em 1997 alguns autores ${ }^{(15)}$, recomendaram a hipocorreção de $20 \%$ da biometria para emetropia em crianças menores de um ano, e de $10 \%$ entre dois e três anos. A conduta adotada no Serviço de Córnea e Catarata do Hospital São Geraldo - UFMG, em crianças até dois anos de idade utiliza-se hipocorreção de $20 \%$, e de 2 a 8 anos de idade hipocorreção de $10 \%$. No Hospital Oftalmológico de Brasília utilizamos geralmente como conduta o implante de uma LIO que possibilite um valor refracional final próximo ao do olho contralateral ou no máximo uma hipocorreção de $10 \%$ da biometria para emetropia.

No nosso paciente, foi realizada cirurgia de catarata com facoaspiração via córnea clara associada a capsulorrexe ante- rior e posterior, vitrectomia anterior central, e optado pelo implante de duas LIO dobráveis "piggyback", dentro do saco capsular.

O uso de duas ou mais lentes para atingir um alto poder dióptrico é opticamente superior ao uso de uma lente única de mesmo poder caso os centros estejam alinhados. Geralmente o limite de poder das lentes produzidas não ultrapassa 34,00 D, visto a alta incidência de aberrações esféricas nestas lentes ${ }^{(16)}$. No presente caso a lente para emetropia seria de 46,50 D. Foram escolhidas duas lentes dobráveis, com poder total de 42,00 D, correspondendo a aproximadamente uma hipocorreção de $10 \%$. O objetivo do implante duplo neste caso foi possibilitar futuramente a retirada de apenas uma das lentes na tentativa de manter uma boa acuidade visual sem correção. A segunda LIO implantada no nosso paciente foi de silicone exatamente para facilitar o seu explante. A utilização de duas lentes de acrílico está relacionada a uma maior incidência de 


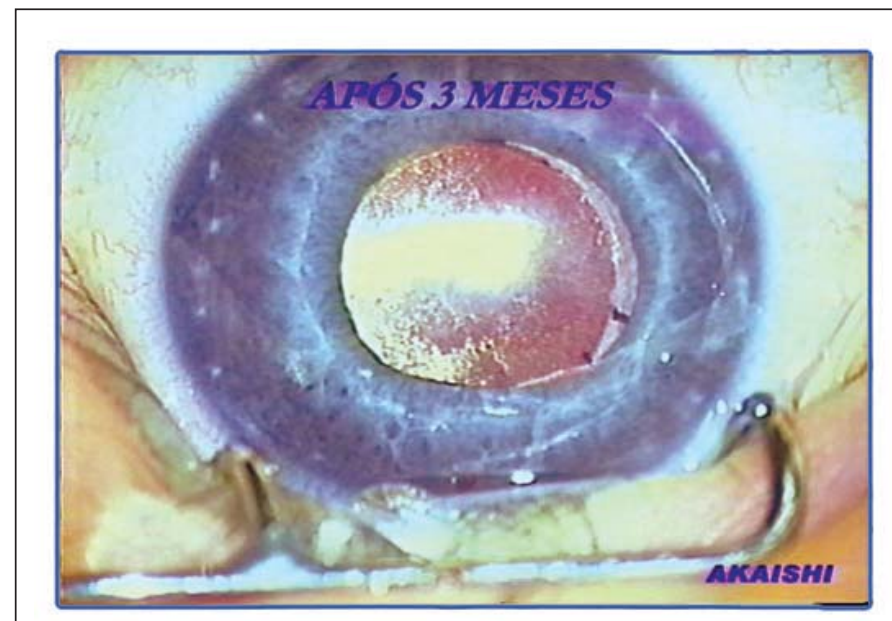

A

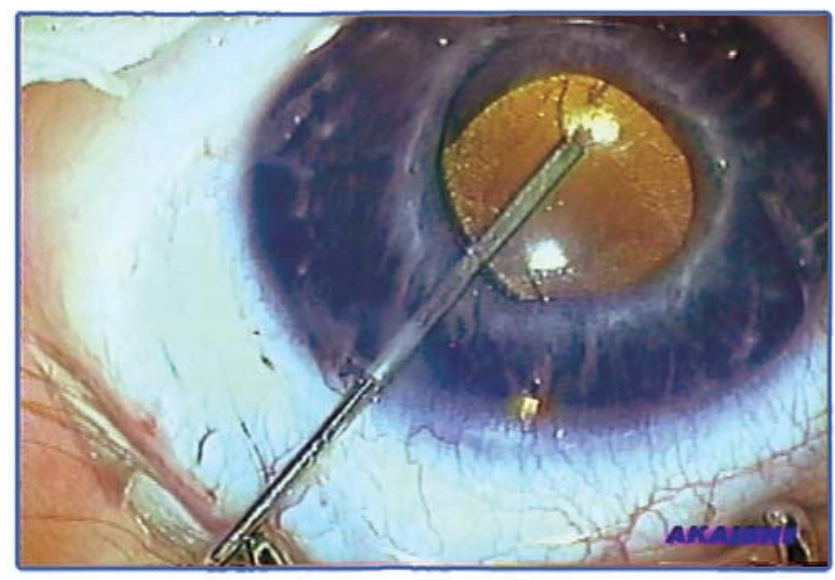

C

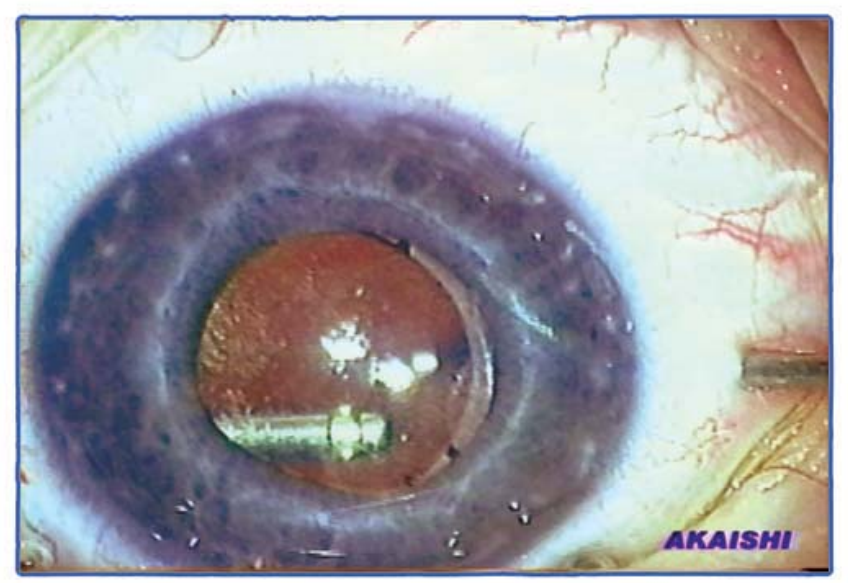

B

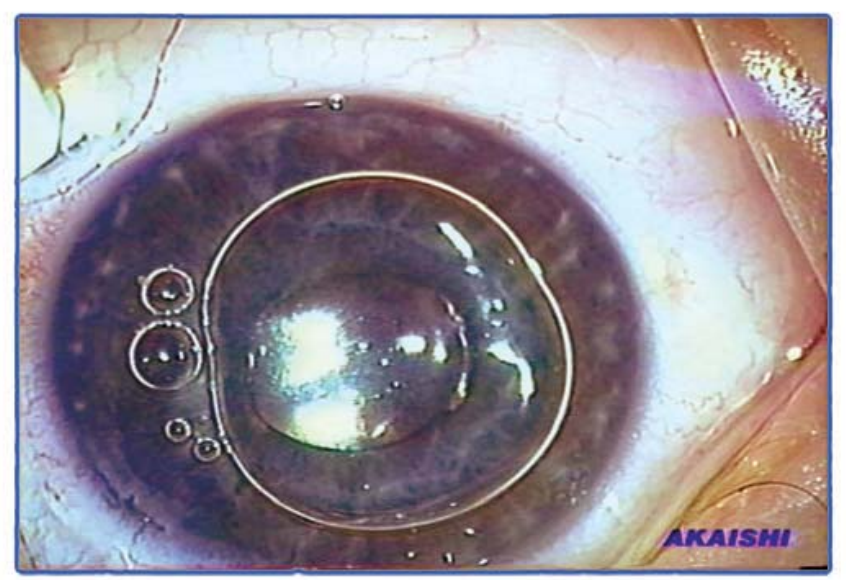

D

Figura 2 - (Akaishi) A: Opacificação de cápsula posterior e do espaço interlenticular após 3 meses de cirurgia; B: Vitrectomia anterior ampla via pars plana; C: Aspiração do material interlenticular; D: Colocação de uma bolha de ar em câmara anterior ao final do procedimento

fibrose e adesão entre as mesmas dificultando ou mesmo impedindo a retirada de apenas uma das lentes ${ }^{(13-14,17)}$.

O implante duplo "piggyback" em crianças com catarata congênita foi descrito pela primeira e única vez em $2001^{(18)}$. Neste trabalho, foram realizados 15 implantes de "piggyback" em 11 crianças entre 16 dias de vida até 6,8 meses de vida. A taxa de reoperação por complicações foi de $26 \%$ nesses olhos sendo estatisticamente sem significância quando comparada a taxa de $22 \%$ nas crianças que receberam apenas uma lente intra-ocular no primeiro ano de vida.

Alguns autores publicaram em 1999 uma taxa de reoperação por complicações após implante de apenas uma lente intra-ocular em crianças com até seis meses de vida de $72 \%$. Essas complicações incluíam glaucoma de ângulo aberto ou por bloqueio pupilar, formação de membrana pupilar e corectopia ${ }^{(19-20)}$.

Além destas, duas outras complicações sabidamente conhecidas do implante de duas ou mais LIOs "piggyback" são: mudanças refracionais e formação de opacificações entre as lentes ${ }^{(13)}$. Na literatura já foram descritas mudanças refracionais em pacientes submetidos a implante duplo de lentes de silicone $^{(5)}$. O desenvolvimento de significativa hipermetropia em seis olhos de três pacientes após implante duplo de lentes de acrílico (4 olhos) e PMMA (2 olhos) já foi descrito ${ }^{(21)}$. Todos os olhos apresentavam proliferação de pérolas de Elschning na interface da parte óptica das LIOs. De acordo com os autores ${ }^{(21)}$, o material localizado no espaço entre as lentes seria responsável por um deslocamento posterior da lente posterior.

A formação de opacidades entre as lentes parece ser agravada quando todas as lentes são implantadas dentro do saco capsular. A verdadeira composição do material entre as lentes ainda não foi completamente estabelecida, restos de material cortical, material viscoelástico, precipitados protéicos e de cálcio já foram identificados entre as lentes ${ }^{(21)}$. Quando as lentes são todas implantadas no saco capsular e a borda da capsulorrexe encontra-se em contato com a superfície anterior da LIO, todas as lentes são seqüestradas no saco capsular. 
Neste cenário, as células epiteliais equatoriais apresentam acesso direto ao espaço interlenticular podendo favorecer a opacificação, fato observado neste relato de caso. Existe uma tendência atual em se implantar uma lente no saco capsular e outra no sulco. Porém por nosso paciente se tratar de um recém-nascido e as possíveis complicações relacionadas ao implante de uma LIO no sulco, principalmente em olhos de pequeno diâmetro, foi optado pelo duplo implante no saco capsular.

Um outro método que poderia ter sido utilizado para reabilitação óptica deste paciente seria o implante de apenas uma lente intra-ocular e a correção óptica através de lente de contato. Vários trabalhos na literatura vêm apresentando bons resultados com essa forma de tratamento ${ }^{(2-3,22)}$. Entre as várias vantagens das lentes de contato estão: a boa qualidade visual, boa indicação em casos de nistagmo e a utilização de poder de acordo com o crescimento ocular. Entre as desvantagens: o alto custo (perda da lente), dificuldade de manuseio e infecções. Quando estas informações foram apresentadas e discutidas com os pais da criança no presente caso, a opção foi pelo implante duplo "piggyback".

Esperamos com o presente trabalho estar contribuindo para o conceito de polipseudofacia ou "piggyback" para o tratamento de catarata congênita. Não temos certeza do impacto do implante de LIOs na acuidade visual final deste paciente. Um maior número de casos e um maior seguimento se farão necessários para se permitir que novos conhecimentos sejam adicionados aos atuais e obter maiores conclusões. Enquanto não se realiza um estudo controlado e randomizado, a decisão de se implantar uma LIO em recém-nascidos deve ser decidida com cuidado e precaução. Em geral as cataratas congênitas possuem prognóstico visual pior que a dos adultos. Sendo que o mesmo dependerá da precocidade do diagnóstico, correta intervenção terapêutica instituída e tratamento da ambliopia.

\section{ABSTRACT}

The authors report a case of congenital cataract, which was treated with piggyback foldable intraocular lens implant, not described in the Brazilian literature. The differences and similarities are analyzed with other reported cases in the literature.

Keywords: Cataract/congenital; Cataract extraction; Intraocular lens; Lens implantation, intraocular; Visual acuity; Case report

\section{REFERÊNCIAS}

1. American Academy of Ophthalmology. Pediatric Ophthalmology and Strabismus, San Francisco: 1997; section 6, part 8 83-95.

2. Buckley E. Pediatrics cataracts and lens anomaly in: Nelson, LB. Harley's pediatric ophthalmology. $4^{\text {th }}$ ed. Philadelphia: W.B. Saunders; 1998. p.258-82.

3. Tartarella MB, Nakano K, Castro CTM, Martins AP. APM. Visão subnormal em crianças. Arq Bras Oftalmol. 1991;54(5):221-4.

4. Adam Netto A, Peres SO. Catarata na infância: Estudo de 106 casos. Rev Bras Oftalmol. 1998;57(12):903-8.

5. Gayton JL, Sanders VN. Implanting two posterior chamber intraocular lenses in a case of microphthalmos. J Cataract Refract Surg. 1993;19(6):776-7.

6. Kora Y, Inatomi M, Fukado Y, Marumori M, Yaguchi S. Long term study of children with implanted intraocular lenses. J Cataract Refract Surg. 1991;18 (5):485-8.

7. Wilson ME, Bluestein EC and Wang XH. Current trends in the use of intraocular lenses in children. J Cataract Refract Surg. 1994;20(6):579-83.

8. Basti S, Ravisshankar U, Gupta S. Results of a prospective evaluation of three methods of management of pediatric cataract. Ophthalmology. 1996;103(3): 713-20.

9. Kara-José N, Arieta CEL. Catarata In: Rodrigues MLV. Oftalmologia clínica. Rio de Janeiro: Cultura Médica; 1992. p.288-302.

10. Hiles DA, Hered RW. Modern intraocular lens implants in children with new age limitations. J Cataract Refract Surg. 1987;13(5):493-7.

11. Wilson ME. Management of aphakia in childhood. Focal Points 1999;17: $1-16$.

12. Katina JH, Lyra JMAG, Souza CD, Trindade FC. Tratamento cirúrgico da catarata pediátrica. Arq Bras Oftalmol. 2002;65(2):193-7.

13. Delmarcelle Y, François J, Goes F, Collingnon-Brach J, Luyckx-Bacus J, Verbraeken H. Clinical ocular biometry (oculometry). Bull Soc Belge Ophtalmol. 1976;172(1):1-608. Francese.

14. Lambert SR, Buckley EG, Plager DA, Medow NB, Wilson ME. Unilateral intraocular lens implantation during the first six months of life. J AAPOS. 1999; 3(6):344-9.

15. Dahan E, Drusedau MU. Choise of lens and dioptric power in pediatric pseudophakia. J Cataract Refract Surg. 1997;23(Suppl 1):618-23

16. Holladay JT, Gills JP, Leidlein J, Cherchio M. Achieving emmetropia in extremely short eyes with two piggyback posterior chamber intraocular lenses. Ophthalmology. 1996;103(7):1118-23.

17. Werner L, Shugar JK, Apple DJ, Pandey SK, Escobar-Gomez M, Visessook N, Evans BB. Opacification of piggyback IOLs associated with an amorphous material attached to interlenticular surfaces. J Cataract Refract Surg. 2000; 26(11):1612-9.

18. Wilson ME, Peterseim MW, Englert JA, Lail-Trail JK, Elliot LA. Pseudophakia and polypseudophakia in the first year of life. JAAPOS. 2001;5(4): 238-45.

19. Lundvall A, Zetterstrom C. Complications after early surgery for congenital cataracts. Acta Ophthalmol Scand. 1999;77(6):677-80.

20. Flitcroft DI, Knight-Nanan D, Bowell R, Lanigan B, O’Keefe M. Intraocular lenses in children: changes in axial length, corneal curvature, and refraction. Br J Ophthalmol. 1999;83(3):265-9.

21. Shugar JK, Schwartz T. Interpseudophakos Elschning pearls associated with late hyperopic shift: a complication of "piggyback" posterior chamber intraocular lens implantation. J Cataract Refract Surg. 1999;25(6):863-7.

22. Ma JJ, Morad Y, Mau E, Brent HP, Barclay R, Levin AV. Contact lenses for the treatment of pediatric cataracts. Ophthalmology. 2003;110(2):299-305. 